\title{
Caso Clínico: Agenesia pericárdica, diagnóstico imagenológico de una patología infrecuente
}

Guillermo Aguilera $S^{1^{*}}$, Velimir Skoknic $B^{1}$, Javiera Aguirre $F^{2}$, Julia Alegría $B^{2}$.

1. Residente de Radiología. Departamento de Imágenes, Facultad de Medicina, Clínica Alemana-Universidad del Desarrollo. Santiago, Chile.

2. Radióloga. Departamento de Imágenes, Facultad de Medicina, Clínica Alemana-Universidad del Desarrollo. Santiago, Chile.

Clinical case: Pericardial agenesis, radiological diagnosis of a rare disease

\section{Resumen:}

La agenesia pericárdica congénita es una condición infrecuente, que generalmente cursa de forma asintomática y es diagnosticada como hallazgo radiológico. Sin embargo, se describe la asociación con malformaciones cardiacas y herniaciones del miocardio con el subsiguiente riesgo de muerte súbita. La radiografía de tórax es un pilar fundamental para la sospecha diagnóstica. Presentamos un caso clínico de una paciente de 11 años que consulta por dolor torácico y disnea, que frente a los hallazgos típicos en radiografía de tórax se sospecha agenesia pericárdica, posteriormente confirmada con TC y RM.

Palabras clave: Agenesis; Diagnostic Imaging; Pericardium.

\begin{abstract}
:
Congenital pericardial agenesis is an infrequent but usually asymptomatic condition, and is diagnosed as an incidental radiological finding. However, the association with cardiac malformations and myocardial herniation with the subsequent risk of sudden death has been reported. Chest plain films are a fundamental tool to raise the diagnostic suspicion. We present a clinical case of an 11-year-old patient who consulted for chest pain and dyspnea, in which, with the typical findings on chest radiography, pericardial agenesis was suspected and later confirmed by CT and MRI.
\end{abstract}

Keyword: Agenesis; Diagnostic Imaging; Pericardium.

Aguilera G., et al. Caso Clínico: Agenesia pericárdica, diagnóstico imagenológico de una patología infrecuente. Rev Chil Radiol 2020; 26(2): 72-75.

*Correo electrónico: Guillermo Aguilera Silva / g.aguilera06@gmail.com

Trabajo enviado el 14 de octubre de 2019. Aceptado para publicación el 17 de marzo de 2020.

\section{Introducción}

La agenesia pericárdica es una condición infrecuente, la cual puede ser adquirida o congénita; esta última, presenta una prevalencia de entre un $0,002 \%-0,004 \%{ }^{(1)}$ según reportes de series quirúrgicas y anatomía patológica. Existe una leve predilección en hombres, con una relación 3:1 entre hombres y mujeres.

El defecto puede estar presente de forma total o parcial, siendo variable el grado de ausencia del pericardio. Es importante reconocer la importancia de los defectos parciales, ya que se ha visto que estos pueden ser causa de una muerte súbita. Los defectos totales normalmente cursan de forma asintomática, por lo que son de menor relevancia clínica(2). Se ha descrito que un porcentaje de ellos podría asociarse a otras malformaciones cardiacas ${ }^{(3)}$. 


\section{Presentación del caso}

Paciente de sexo femenino de 11 años, con historia de infecciones respiratorias recurrentes en la infancia, que consulta por un cuadro de 6 meses de evolución de dolor torácico atípico de leve intensidad y disnea, por lo que se le solicita radiografía de tórax, electrocardiograma y ecocardiografía. Los exámenes de laboratorio generales no demostraron ninguna alteración significativa. El electrocardiograma demostró desviación del eje cardiaco a derecha, sin otras alteraciones. No fue posible realizar el ecocardiograma por falta de una ventana adecuada para evaluar las estructuras cardiacas, por lo que se solicitó una tomografía computada de tórax contrastada para evaluar la anatomía torácica.

La radiografía de tórax PA (Figura 1) demuestra importante desviación de la silueta cardiaca hacia el hemitórax izquierdo, con el ápex orientado hacia el ángulo costofrénico ipsilateral y levemente rotado hacia posterior, con aparente elongación del reborde del ventrículo izquierdo, aumento de la silueta de la arteria pulmonar y aumento relativo del espacio de la ventana aortopulmonar. No se identificaron alteraciones del parénquima ni desviación de la alineación traqueal.

En la tomografía computada (TC) en cortes axial y coronal (Figuras 2 y 3 ) se observa una marcada levoposición del corazón, con un segmento de parénquima pulmonar de la língula interpuesto entre la aorta ascendente y el tronco de la arteria pulmonar. Las restantes estructuras del mediastino y el parénquima pulmonar no presentaron alteraciones, en especial no se observaron malformaciones ni variantes anatómicas. El estudio presentaba artefacto de movimiento, por lo que no era posible determinar la presencia de pericardio. Ante la alta sospecha radiológica de agenesia pericárdica, y para evaluar funcionalidad cardiaca y descartar otras alteraciones asociadas, se realizó una resonancia magnética (RM) de corazón.

En imágenes axiales de la RM (Figura 4), se observa el corazón en íntima relación con la pared torácica izquierda, sin identificar presencia de pericardio; compatible con una agenesia total. No se encontraron alteraciones dinámicas ni variantes de la anatomía cardiaca en el estudio.

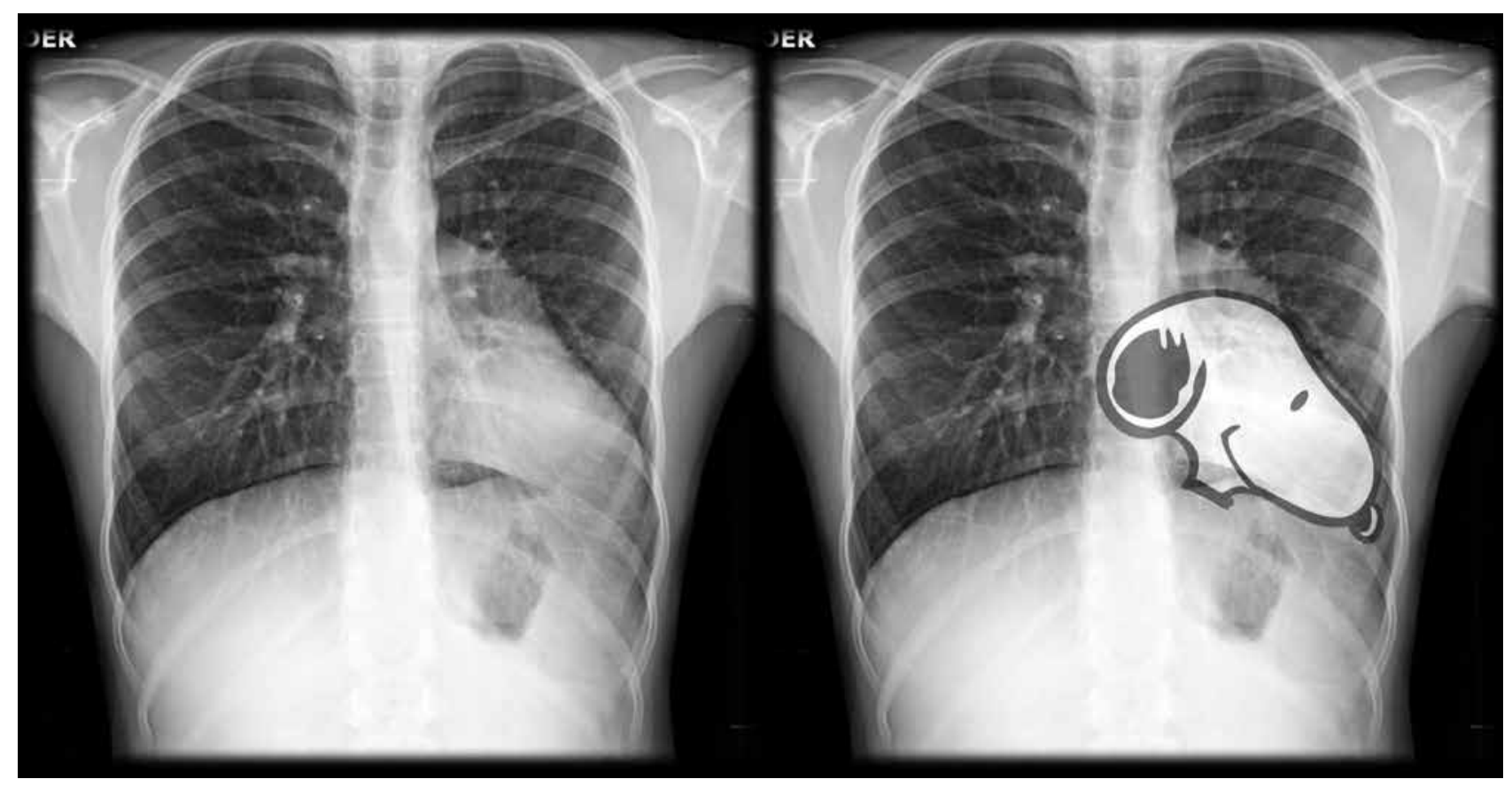

Figura 1: Radiografía de tórax $P A$, con marcada desviación de la silueta cardiaca hacia la izquierda. La tráquea se encuentra en línea media. El tronco de la arteria pulmonar impresiona aumentado de tamaño. El ventrículo izquierdo se observa aplanado y alargado. Estos hallazgos dan la clásica apariencia del personaje infantil "Snoopy". 


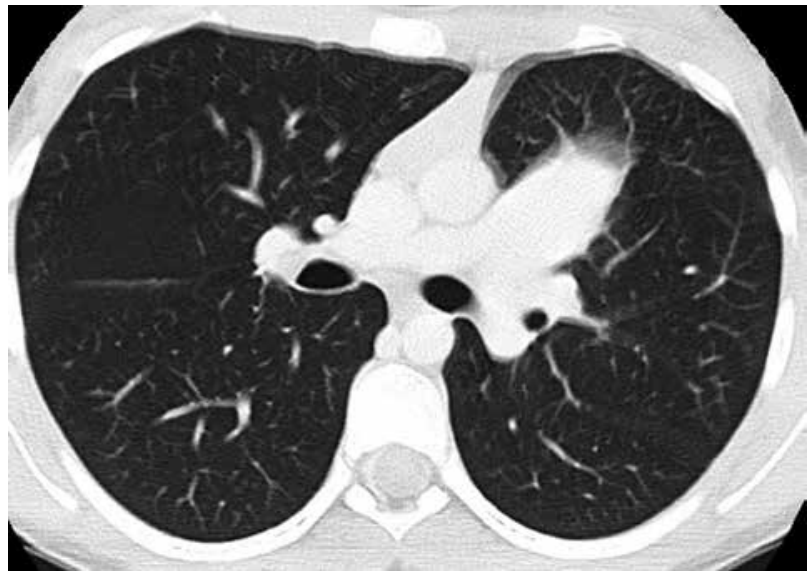

Figura 2: Corte axial de tomografía computada de tórax, algoritmo pulmón. Se observa interposición de parénquima pulmonar entre la aorta ascendente y el tronco de la arteria pulmonar. No se observan alteraciones del parénquima.

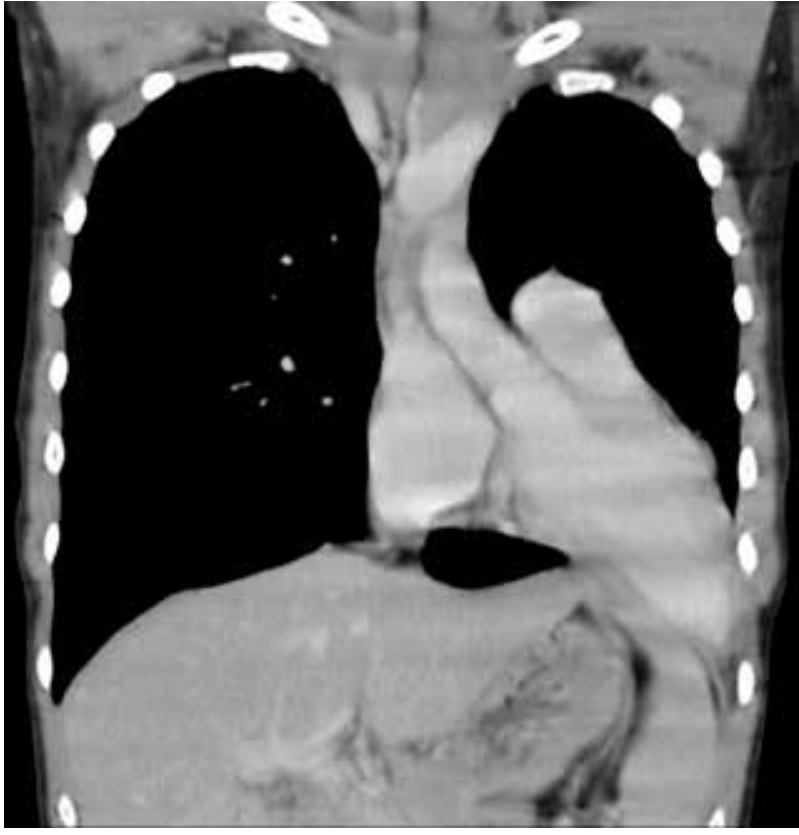

Figura 3: Reconstrucción coronal de tomografía computada tórax con contraste, ventana mediastínica. Levo-posición cardiaca con ápex orientado hacia el ángulo costofrénico. Origen normal de los grandes vasos mediastínicos.

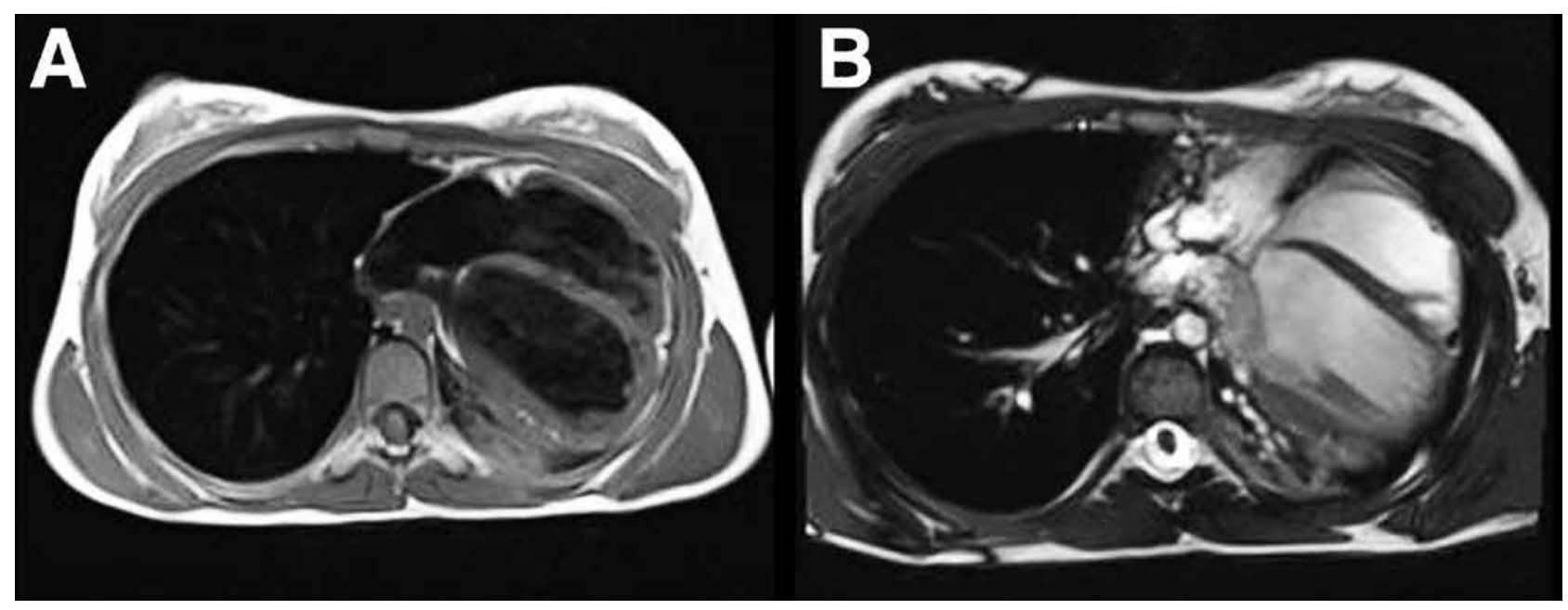

Figura 4: Resonancia magnética cardiaca. T1 TSE axial (A) y SSFP axial (B), que demuestran ausencia total de pericardio; confirmando el diagnóstico. Restos de los hallazgos en concordancia con los estudios previos.

\section{Discusión}

Las agenesias pericárdicas plantean un desafío diagnóstico para el radiólogo, ya que presentan una baja frecuencia y una sintomatología específica, por lo que muchas veces es subdiagnosticada.

La radiografía de tórax juega un rol fundamental en la pesquisa y sospecha de esta condición, tal como fue descrito en el caso clínico (Figura 1), la mayoría de los casos de agenesia pericardio parcial izquierdo o total, siendo la primera más frecuente, representando un $70 \%$ y $9 \%$ de los casos respectivamente ${ }^{(4,5)}$. Puede observarse extrema desviación del corazón hacia el hemitórax izquierdo, con aplanamiento y elongación del ventrículo izquierdo, interposición de parénquima pulmonar en la ventana aorto-pulmonar y pérdida de 
la definición del borde cardíaco derecho por superposición con la columna, lo que da una imagen similar a la cabeza de "Snoopy" (Figura 1)(6,7). Es significativo destacar que no existe desviación traqueal asociada, lo cual ayuda a descartar otras alteraciones del parénquima pulmonar o del hemitórax ${ }^{(1)}$.

La tomografía computada, exhibe los mismos hallazgos descritos, siendo uno de los signos más importantes la desviación del corazón a izquierda y la interposición de parénquima pulmonar entre la aorta ascendente y el tronco de la arteria pulmonar. Cabe destacar que, siendo la TC un examen útil, el mejor estudio para confirmar la ausencia de pericardio es la RM cardiaca; donde sumado a lo anterior, habrá ausencia de la fina banda de tejido hipointenso en T1 y T2 que recubre el corazón. Además, es importante al aporte funcional y anatómica que entrega la RM, sobre todo ante la imposibilidad de realizar estudio con ecocardiograma, como en nuestro caso, y el hecho de que no se necesita contraste, ni irradia. En casos de agenesia completa los ligamentos pericárdicos que fijan el pericardio al mediastino y diafragma no están presentes.

En defectos totales el electrocardiograma típicamente demuestra desviación del eje cardiaco a derecha con o sin bloqueo de rama derecha y bradicardia ${ }^{(5)}$.

Se ha reportado que hasta un $30 \%$ de estos pacientes presentan distintas anomalías cardiacas asociadas, dentro de las más frecuentes e importantes se encuentran el ductus arterioso persistente, defecto septal atrial, tetralogía de Fallot, estenosis mitral o prolapso, y válvula aortica bicúspide; también hay descrito casos con quistes broncogénicos, hernias diafragmáticas y secuestros pulmonares e incluso se reportan anomalías de la pared torácica, como el pectum excavatum ${ }^{(8,9)}$.

Las manifestaciones clínicas son variadas. La mayoría de los casos cursan de forma asintomática, otras, con síntomas inespecíficos como dolor torácico atípico, disnea o mareos ${ }^{(3)}$. Los defectos parciales son los que revisten mayor relevancia, ya que requieren tratamiento quirúrgico para su manejo, debido a que existe riesgo reportado de la herniación de parénquima miocárdico a través del defecto, con consiguiente isquemia, atrapamiento de los músculos papilares de la válvula tricúspide, con posterior insuficiencia de ésta ${ }^{(1)}$. Los defectos totales, o defectos de pequeño tamaño, no revisten mayores complicaciones y no requieren un tratamiento quirúrgico.

\section{Conclusiones}

La agenesia pericárdica es una patología congénita poco frecuente con clínica inespecífica, en la que el conocimiento de los signos en la radiografía es fundamental para su sospecha, siendo el más clásico de éstos el signo de la cabeza de "Snoopy".

\section{Referencias}

1. Abbas AC. Congenital absence of the pericardium: Case presentation and review of literature. Int $\mathrm{J}$ Cardiol. 2005; 98(1): 21-25.

2. Ankit $B$. Shah IK. Congenital defects of the pericardium: A review. European Heart Journal. 2005; 16(8): 821-827.

3. Han XH. Snoopy sign: Congenital absence of the left pericardium. J Med Imaging Radiat Oncol. 2018; 62: 47-47.

4. Kim CY. Congenital Absence of the Pericardium. J Cardiovasc Ultrasound. 2014; 22(1): 36-39.

5. Montaudon RF. Congenital pericardial defect: report of two cases and literature review. Surg Radiol Anat. 2007; 29(3): 195-200.

6. Gatzoulis MA., Munk MD., Merchant N., Van Arsdell, GS., McCrindle BW., Webb GD. Isolated congenital absence of the pericardium: clinical presentation, diagnosis, and management. The Annals of thoracic surgery. 2000: 69(4): 1209-1215.

7. Panagiotopoulos BJ. The Snoopy sign. European Journal of Cardio-Thoracic Surgery. 2013; 45(3): 586586.

8. Psychidis-papakyritsis DR. Functional MRI of congenital absence of the pericardium. AJR Am J Roentgenol. 2007; 189(6): 312-314.

9. Yamano ST. Magnetic resonance imaging differentiated partial from complete absence of the left pericardium in a case of leftward displacement of the heart. Circ J. 2004; 68(4): 385-388. 